\title{
Group Support Interventions for Women With Breast Cancer: Who Benefits From What?
}

\author{
Vicki S. Helgeson and Sheldon Cohen \\ Carnegie Mellon University
}

\author{
Richard Schulz \\ University of Pittsburgh
}

\author{
Joyce Yasko \\ University of Pittsburgh School of Nursing and University of Pittsburgh Cancer Institute
}

\begin{abstract}
Research on the benefits of social support groups has been inconclusive. One reason is that individual differences in intervention responses have rarely been examined. The authors determined the extent to which individual difference variables moderated the effects of an information-based educational group and an emotion-focused peer discussion group on the mental and physical functioning of women with breast cancer $(n=230)$. The authors administered the SF-36 (S. E. Ware, K. K. Snow, M. Kosinski, \& B. Gandek, 1993), a multidimensional quality of life instrument, pre- and postintervention. Educational groups showed greater benefits on the physical functioning of women who started the study with more difficulties compared with less difficulties (e.g., lacked support or fewer personal resources). Peer discussion groups were helpful for women who lacked support from their partners or physicians but harmful for women who had high levels of support. Implications of these results for clinical interventions are discussed.
\end{abstract}

Key words: support groups, cancer, individual differences

Despite the prevalence of peer support groups in the community, the evidence for their effects on well-being are mixed at best. Support groups for people with cancer (Helgeson \& Cohen, 1996), caregivers (Bourgeois, Schulz, \& Burgio, 1996; Lavoie, 1995), people facing the transition to parenthood (Cowan \& Cowan, 1986), people recently divorced (Hughes, 1988), and people who have been victimized (Coates \& Winston, 1983) do not show clear benefits. We define support groups as groups that contain group discussion or information exchange that is led by a trained facilitator. In other words, we distinguish support groups from self-help groups, which do not have an expert leader.

One reason that strong benefits of support groups may not have appeared is that investigators have rarely examined

Vicki S. Helgeson and Sheldon Cohen, Department of Psychology, Carnegie Mellon University; Richard Schulz, Department of Psychiatry, University of Pittsburgh; Joyce Yasko, University of Pittsburgh School of Nursing and University of Pittsburgh Cancer Institute.

The results of this research were presented at the Society of Behavioral Medicine meeting in New Orleans, March 1998.

This research was funded by National Institutes of Health (NIH) Grant R01 CA61303 and by NIH Senior Scientist Award MH00721. We are grateful to the facilitators and speakers who conducted the groups as well as to over 40 physicians who referred patients to the study. We also thank Pamela Blair, Laura Bogart, Antonina D. Karaczun, Karen Pealer, and Pamela Snyder for their invaluable assistance with this research.

Correspondence concerning this article should be addressed to Vicki S. Helgeson, Department of Psychology, Carnegie Mellon University, Pittsburgh, Pennsylvania 15213-3890. Electronic mail may be sent to vh2e+@@andrew.cmu.edu. individual differences in responses to the intervention. To be fair, this is not the goal of randomized clinical trials; the intent is to minimize the variance attributable to individuals. Nonetheless, it is possible that some individuals benefit from an intervention, some are unaffected, and some could even be harmed. The goal of this article is to examine the extent to which individual difference variables moderate the effect of support-group interventions on psychological and physical functioning for people with cancer.

Although numerous reviews of the literature on interventions for people with cancer are aimed at determining what kind of intervention is most effective (Andersen, 1992; Fawzy, Fawzy, Arndt, \& Pasnau, 1995; Helgeson \& Cohen, 1996; Meyer \& Mark, 1995), rarely do these reviews address the question of what kind of person benefits from which kind of intervention. Some authors suggest that it is important to take psychological variables into account, such as baseline functioning, when examining the impact of an intervention (Trijsburg, van Knippenberg, \& Rijpma, 1992), but the suggestion is that these variables are statistically controlled rather than examined as moderators of an intervention's effect. The awareness that all kinds of people might not equally benefit from a support-group intervention certainly exists. However, only a few specific predictions about moderation have been made.

For example, Andersen (1992) suggested that the level of support from family and friends might moderate the effectiveness of an intervention. People with more problematic social relations might benefit the most from a peer support-group intervention. If true, this theory might explain why some support-group studies fail to find positive effects. For 
example, Wandersman (1982) suggested that one reason support-group interventions for new parents have had null effects is that the majority of parents are satisfied with their social network relations.

In the case of cancer, there is reason to believe that patients have some problems with their social networks. Network members do not always behave in supportive ways. For example, network members do not always allow the patient to express distress over the illness because they believe such expressions are unhealthy or because such expressions make them feel uncomfortable. Network members may force the patient to be cheerful, minimize the patient's problems, and withdraw from social interaction (Dakof \& Taylor, 1990; Dunkel-Schetter, 1984; PetersGolden, 1982). All of these behaviors can be construed as failures to provide emotional support (Helgeson \& Cohen, 1996). One of the rationales behind the peer support group is that peers are unlikely to behave in these ways and may be able to compensate for support deficits from the natural network. Yet, one should not assume that all interactions with network members are negative. The majority of people with cancer report very supportive and satisfying network relations (Dakof \& Taylor, 1990; Neuling \& Winefield, 1988). In sum, there may be variability in network relations among people with cancer such that those with support deficits benefit more from the peer support group than those without such deficits.

In general, it is thought that support interventions ought to be directed at those who are at highest risk for adverse outcomes (Watson, 1983). The high-risk group may consist of individuals who lack social support and/or lack personal resources, such as self-esteem, to cope with a stressor. One study of people with cancer supported the latter claim by showing that only individuals who were low in ego strength benefited from an individual coping skills intervention (Edgar, Rosberger, \& Nowlis, 1992).

The goal of the present article is to examine whether certain people were more likely to benefit from one kind of support intervention than another. To address this goal, we drew on data from a study that evaluated the effects of two kinds of support-group interventions for people with cancer: education and peer discussion. Briefly, women with newly diagnosed breast cancer were randomly assigned to one of four conditions: education group intervention, peer-discussion group intervention, education plus peer-discussion group intervention (combined), and control group. Thus, a 2 (education) $\times 2$ (peer discussion) factorial design was used. One of the strengths of this study was that seven separate groups were conducted in each of the four conditions. Each group was composed of 8-12 women. Although we predicted an interaction between education and peer discussion, such that the combined condition would show the best adjustment, we only found main effects of the education intervention on psychological and physical well-being (Helgeson, Cohen, Schulz, \& Yasko, 1999). That is, conditions with education showed superior adjustment relative to conditions without education. There was no effect of peer discussion on well-being. The intervention effect and mecha- nisms describing effects are reported in Helgeson et al. (1999).

We now ask whether certain subgroups of women were most likely to benefit from the education intervention and whether we can identify a subset of women who benefited from the peer-discussion intervention. It is possible that the education intervention's benefits are limited to a subgroup of women or are stronger for a subgroup of women. The null effects of the peer discussion group may be due to benefits being conferred on some individuals and harm being conferred on others.

Because it is not clear how the two components (education and peer discussion) interacted in the combination condition, we address the issue of who benefits from what by focusing on the education only and the peer discussion only interventions. This focus simplified our analyses and reduced the number of statistical tests performed. Because the education and peer discussion interventions had such different effects on outcomes (Helgeson et al., 1999), it may not be possible to understand their synergistic effects in the combined condition in this study.

\section{Predictions: Who Benefits From What?}

The peer-discussion intervention was aimed at providing emotional support. The focus of the intervention was on the sharing of experiences and expression of feelings. Facilitators were expected to guide the discussion so that group members' experiences would be normalized and feelings validated. We expected that people who reported at baseline that they lacked emotional support from their partners and/or reported more negative interactions with their partners (which can be construed as the failure to provide emotional support) would benefit from peer discussion.

The educational intervention was aimed at providing informational support. The focus of this intervention was on providing women with information they could use to understand breast cancer, to manage their treatment side effects, and to enhance their overall recovery. This is the kind of information we expect women to receive from their physician. Thus, we predicted that people who reported that they lacked informational support from their physician when entering the study would be most likely to benefit from the education intervention.

We also tested whether there were individual differences in personal resources, such as self-esteem or personal control, that influenced who benefited from an intervention. Specifically, we tested whether people who lacked personal resources at the outset of the study would be more likely to benefit from a particular intervention. In a previous report, it was shown that the educational intervention increased self-esteem, body image, and perceived control and reduced uncertainty about the illness (Helgeson et al., 1999). These variables can be viewed as personal resources that women possessed or lacked at the start of the intervention. The education intervention was organized around several of these resources. The theme of the intervention was control; that is, we were providing women with information to enhance their control over the illness experience. The 
information provided in the education group was expected to reduce people's sense of confusion or uncertainty regarding their illness. Thus, we predicted that the educational intervention would be most beneficial to women who lacked personal resources.

\section{Method}

\section{Participants}

Women were eligible to participate if they had been diagnosed with Stage I or II breast cancer and were treated with surgery and adjuvant chemotherapy. We contacted 445 women. Of these, 364 (82\%) agreed to be interviewed and $312(70 \%)$ agreed to the randomization. Of the 81 women who refused any participation, 55 provided some demographic and baseline quality of life information over the telephone. A comparison of participants $(n=312)$ to nonparticipants $(n=107[55+52])$ revealed that those who refused randomization were more likely to be married, were less educated, and were less likely to have attended any lectures on breast cancer (all $p s<.05$ ). There also was a trend for nonparticipants to report worse health behavior than participants $(p<.10)$, further suggesting that nonparticipants were less interested in health issues. There were no differences between the two groups on baseline quality of life outcomes.

Our review of medical records revealed that women had Stage I $(25 \%)$, Stage II $(69 \%)$, or Stage III $(6 \%)$ breast cancer. The majority of women received lumpectomies $(68 \%)$ rather than mastectomies (32\%), which is consistent with the norms for the Pittsburgh area. Participants' ages ranged from 27 to $75(M=48$, $S D=9.64)$. The majority of women were Caucasian $(93 \%) ; 7 \%$ were African American, and $1 \%$ was Hispanic. Education levels were reported to be $4 \%$, less than high school; $30 \%$, high school graduate; $28 \%$, some college; $24 \%$, college graduate; and $14 \%$, postgraduate training. Income was reported to be less than $\$ 20,000$ (15\%), $\$ 20-30,000(13 \%), \$ 30-40,000(14 \%), \$ 40-50,000(13 \%)$, $\$ 50-75,000(28 \%), \$ 75-100,000(2 \%)$, and greater than $\$ 100,000$ $(5 \%)$. Nine percent $(9 \%)$ of women refused to answer the question.

Women were randomly assigned to a control condition or one of three support group interventions: peer discussion only, education only, and combined (i.e., education plus peer discussion). There were 28 groups, each consisting of 8-12 women: 7 education groups $(n=79), 7$ peer-discussion groups $(n=74), 7$ combination groups $(n=82)$, and 7 control groups $(n=77)$. (For further details about the interventions and their specific effects, see Helgeson et al., 1999.) In the present article, we only contrast the education and peer-discussion conditions with the control condition. The goal of the present report is to determine whether characteristics of patients assessed at baseline predict differences in who benefited from each intervention.

\section{Procedure}

Women were recruited for the study from the offices of over 40 medical oncologists shortly after they began chemotherapy. Nurses provided us with the names and phone numbers of patients who were willing to listen to a description of our research study. Although we have no record of the number of women who refused a phone contact, we are confident that it was rare. The physicians supported the study, and the nurses only requested permission to reveal their phone numbers to us. Because nurses did not provide patients with any information about the study, patients had no basis to decline. The women who agreed to randomization were interviewed in their homes for about $90 \mathrm{~min}$. Recruitment began in
September of 1993 and ended in May of 1996. Women did not know the group to which they would be assigned until after the interview. On average, the intervention began 4 months after diagnosis.

The education and peer-discussion intervention groups met weekly for 8 weeks. Pairs of oncology nurses and oncology social workers (both with master's degrees) facilitated both interventions so that facilitator was not confounded with intervention type. Facilitators had experience conducting support groups for people with cancer. Facilitators attended a 1-day workshop in which the content of the educational and peer-discussion interventions was reviewed and guidelines for conducting the groups were provided. The education intervention consisted of a $45-\mathrm{min}$ presentation by a facilitator or a guest speaker (physician, dietician, or physical therapist) on a topic of relevance to breast cancer and its treatment (e.g., side effects from chemotherapy, nutrition). Homework exercises were used to reinforce the intervention principles. The peer discussion intervention consisted of $60 \mathrm{~min}$ of facilitator-led group discussion. The emphasis was on the sharing of experiences and expression of feelings. Facilitators encouraged the expression of both positive and negative affect.

We tried to make the interventions as distinct as possible. The educational intervention was conducted in a classroom type format. Discussion among the women was discouraged. The peerdiscussion intervention focused on the discussion of feelings and experiences. Facilitators refrained from providing information during the group discussions unless information was necessary to correct an exchange of misinformation among the women.

One to 2 weeks after the intervention, participants in all conditions were interviewed again by phone and mailed questionnaire. Social support from the network and personal resources were measured during the initial interview. The outcome variables, which assess psychological and physical functioning, were measured both pre- and postintervention.

\section{Instruments}

Social support. We developed a 15 -item support scale based on Vaux, Riedel, and Stewart's (1987) measure of perceived availability of support. Patients were provided with a list of ways that people help each other and asked whether their spouse (or partner) would be able to provide each kind of help. Responses were made on a 4 -point scale: $1=$ would not, $2=$ might, $3=$ probably, $4=$ certainly. Five items assessed emotional support (e.g., "How likely would this person be to comfort you if you were upset?"), 5 items assessed informational support (e.g., "How likely would this person be to give you advice about what to do?"), and 5 items assessed instrumental support (e.g., "How likely would this person be to run an errand for you?"). During the initial interview, we administered all three subscales with respect to the woman's husband or partner. Three fourths $(75 \%)$ of the women had partners, so the analyses for these variables are limited to the 58 education participants, the 53 peer discussion participants, and the 60 control participants. The emotional and informational support items were administered with respect to the woman's medical oncologist. Thus, all women were included in these analyses. The reliabilities of all of the support scales were good, with alphas ranging from .70 to .89 .

We developed a 10 -item measure of negative interactions with network members based on research on failed support attempts (Dakof \& Taylor, 1990; Wortman \& Lehman, 1985). ${ }^{1}$ Patients were

${ }^{1}$ A copy of the social support and negative interaction instruments is available from Vicki $S$. Helgeson. 
told that people may have good intentions but sometimes say or do something that upsets them. They indicated the frequency with which their spouse (or partner) engaged in each item on a 5-point scale $(1=$ never; $5=$ very often $)$. Items included not understanding the situation, acting uncomfortable when talking about the illness, changing the subject when trying to discuss the illness, and trivializing problems. The internal consistency was .85 . Many of these items can be construed as the failure to provide emotional support (Helgeson \& Cohen, 1996).

Personal resources. During the initial interview, we administered the following four instruments. We used the Rosenberg Self-Esteem scale (Rosenberg, 1965) to measure global self-esteem and developed a 14-item body-image scale based on the Cancer Rehabilitation Evaluation Systems (CARES; Schag \& Heinrich, 1988 ). Internal consistencies were high ( $\alpha$ s $=.83$ and .89 , respectively). We measured perceived personal control over the illness experience with three items that have been used in previous studies with chronically ill populations (Campbell, Dunkel-Schetter, \& Peplau, 1991; Collins, Taylor, \& Skokan, 1990; Helgeson, 1992). The three items tapped the domains of future course of illness, day-to-day illness symptoms, and emotions related to illness $(\alpha=.70)$. We administered an abbreviated form of the illness ambiguity subscale from Mishel's Uncertainty about Illness Scale (Mishel, 1981; $\alpha=.71$ ). The nine items chosen had the highest loading on Mishel's factor analysis. Because all four of these scales loaded on a single principal component following factor analysis and only one factor could be extracted, we developed a composite index that represented personal resources. We standardized the four scores and summed them.

Outcomes. We used the SF-36 from the Medical Outcomes Study (Ware, Snow, Kosinski, \& Gandek, 1993) to measure health-related quality of life at both the initial and follow-up interview. This instrument has excellent reliability and validity and has been used to evaluate functional status in depressed, chronically ill, and healthy populations (Wells et al., 1989). It contains eight multi-item scales: general health perceptions, physical functioning, role limitations due to physical problems, bodily pain, mental health, vitality, role limitations due to emotional problems, and social functioning. Principal-components analysis, followed by varimax rotation of the 36 items, revealed eight factors with eigenvalues greater than one at both times of assessment. Items loaded on their respective eight scales. The authors have derived factor weights for the eight scales so that a mental health component score (MCS) and a physical health component score (PCS) can be created (Ware, Kosinski, \& Keller, 1995). Weights are assigned to all eight scales to create MCS and PCS. The variables with the highest loading on PCS (in order from highest to lowest) are physical functioning, role limitations due to physical problems, bodily pain, and general health perceptions. The variables with the highest loading on MCS are mental health, role limitations due to emotional problems, social functioning, and vitality. Cronbach's alphas for the scales were high during the initial and follow-up interview (ranged from .80 to .91 ). Higher numbers indicate better functioning.

Pre- to postintervention MCS scores and PCS scores did not change in the control group or the peer-discussion group but did significantly improve $(p<.05)$ in the educational group (see Helgeson et al., 1999, for more detail).

\section{Data Analysis}

We used the intention-to-treat principle (Newell, 1992) in our analyses, which means that we retained all of the women who were randomly assigned to condition in the analysis regardless of their level of attendance. Thus, we used a conservative analysis. Among those who attended, average attendance was six out of the eight meetings. Ten percent of women did not attend any meetings. Attendance was not correlated with either of the outcomes and was only correlated with one predictor variable, oncologist informational support $(r=-.14, p<.05)$.

We used multiple-regression analysis to determine whether individual difference variables interacted with a particular intervention to predict the outcomes. First, we created two dummy variables to represent the comparison between the education intervention and the control group and between the peer-discussion intervention and the control group. Because our hypotheses differed about who would benefit from the educational and peer-discussion interventions relative to the control group, it was important to contrast each intervention with the control group rather than combine the two interventions into an intervention versus control contrast. Next, we created two interaction terms to reflect the interaction of the psychosocial variable with each of the two dummy-coded intervention-control contrasts. Prior to creating the interaction terms, we centered the variables as suggested by J. Cohen and Cohen (1983) to reduce multicollinearity between the independent variable and the interaction term.

The first step of the regression analysis contained the Time 1 measure of the dependent variable so that we could predict changes in dependent measures (MCS, PCS) over time. The second step contained the main effects for the psychosocial variable and the two dummy-coded intervention-control contrasts, and the third step contained the two interaction terms.

Significant interactions were interpreted in two ways. First, we used the procedures suggested by J. Cohen and Cohen (1983). We entered the psychosocial variable in two separate regression equations, one for each of the two conditions (e.g., education condition and control condition) involved in the interaction term. We then plotted the scores of those individuals who scored one standard deviation above and below the mean of the psychosocial variable in each of the two conditions. These plots would show us how the relation of a psychosocial variable to an outcome differed for the two conditions. For example, a psychosocial variable might be more strongly related to an outcome (i.e., have a steeper slope) for one condition compared with another. These plots of residualized change scores, however, make it difficult to determine if people are improving or deteriorating over time in an absolute sense. Thus, we plotted all interactions a second way. For each of the two conditions involved in the interaction, we divided the sample into those who scored high and low on the psychosocial variable at Time 1 using a median split. We then plotted the dependent variable scores of the low group and the high group at Time 1 and Time 2 for the two conditions separately. For example, for an interaction between partner emotional support and peer discussion versus control, we could examine how high- and low-support individuals' adjustment changed over time in the peer support and control conditions. Although the second plots are more crude, in every example reported below the second plots are consistent with the findings from the first plots and more informative about the direction of the change. Thus, we present the second plots in the figures in the article.

\section{Results}

The individual difference variables of interest (partner emotional support, partner negative interactions, oncologist informational support, personal resources index) were mod- 
estly related ( $r$ range from .19 to .32 ), with the exception of partner emotional support and partner negative interactions, which were strongly inversely related $(r=-.73, p<.001)$. There were no significant intervention differences in the individual differences variables or baseline adjustment. Thus, the random assignment was effective with respect to our hypothesized moderator variables and outcome variables. However, we always control for baseline adjustment in our analyses.

Neither education, income, nor stage of disease were associated with individual difference variables or outcome variables. Age was not associated with outcome variables but was associated with one predictor variable, negative partner interactions $(r=-13, p<.05)$. Kind of surgery was only related to one variable, MCS. Women with mastectomies had better mental functioning $(M=48.15)$ than women with lumpectomies $(M=45.73), t(310)=$ $1.97, p=.05$.

\section{Social Resources}

Partner emotional support interacted with the education intervention $(\beta=-1.48, p<.005)$ and the peer-discussion intervention $(\beta=-2.30, p<.001)$ to predict post PCS scores. These interactions are displayed in Figure 1 . Women who lacked emotional support from their partner were buffered by each of the two interventions from the deterioration in physical functioning observed in the control group. However, women who had high emotional support from their partner did not react the same to the two interventions. These women were unaffected by the education intervention but adversely affected by the peer-discussion intervention.

Negative interactions with partner interacted with the education intervention $(\beta=.53, p<.05)$ and the peerdiscussion intervention $(\beta=.53, p<.05)$ to predict post PCS. The interactions with PCS were mirror images of the ones shown in Figure 1. Again, women who reported more negative interactions with their partner were buffered by either of the interventions from the deterioration in physical functioning observed in the control group. Women who had few negative interactions with their partner, however, did not react the same to the two interventions. These women were relatively unaffected by the education intervention but were adversely affected by the peer-discussion intervention.

Oncologist informational support also interacted with the education intervention $(\beta=-.96, p=.06)$ and the peerdiscussion intervention $(\beta=-1.16, p=.01$ ) to predict post PCS. The interactions are shown in Figure 2. The patterns are the same as those identified above. Women who reported low oncologist informational support benefited from either intervention compared with the control group. Women who reported high oncologist informational support were unaffected by the education intervention compared with the control group but were adversely affected by the peerdiscussion intervention compared with the control group.

It is worth noting that no interactions were observed-nor predicted-between either intervention and partner informational or instrumental support or oncologist emotional support. In addition, significant interactions did not appear for post MCS.

\section{Personal Resources}

The personal resources index interacted with the education intervention to predict post PCS $(\beta=-.21, p<.01)$. Personal resources did not interact with the peer discussion intervention on either outcome. As shown in Figure 3, women who lacked personal resources were buffered by the education intervention from the deterioration in physical functioning over time observed in the control group. It also is worth mentioning that this same interaction was significant for three of the variables that composed the personal resources index-personal control $(\beta=-.72, p=.05)$, body image $(\beta=-.84, p<.05)$, self-esteem $(\beta=-1.56$, $p<.01$ - and marginal for one, uncertainty about illness $(\beta=.37, p=.13)$.

\section{Partner Emotional Support}
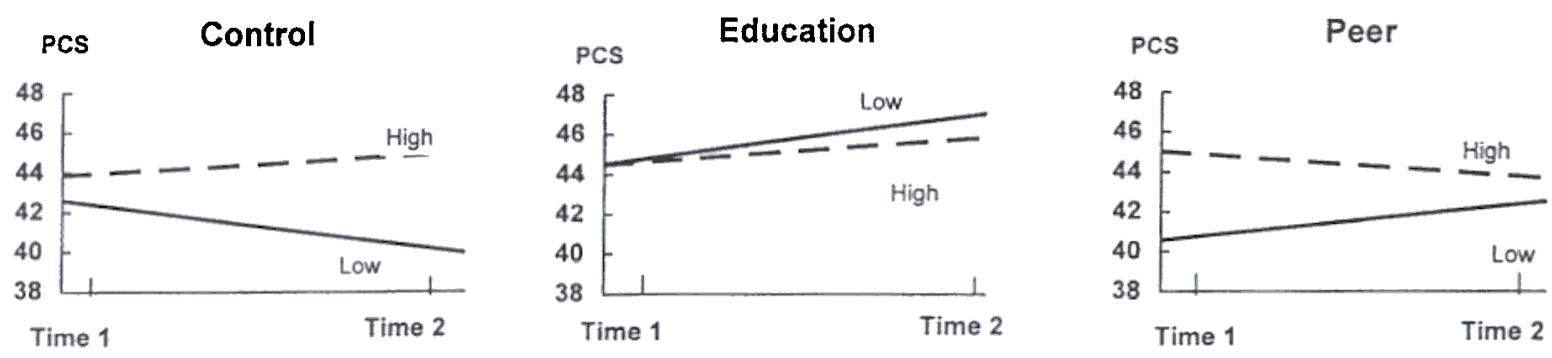

Figure 1. Interaction of baseline partner emotional support with education and peer-discussion interventions on PCS (physical functioning). Higher scores mean better functioning. 


\section{Oncologist Informational Support}
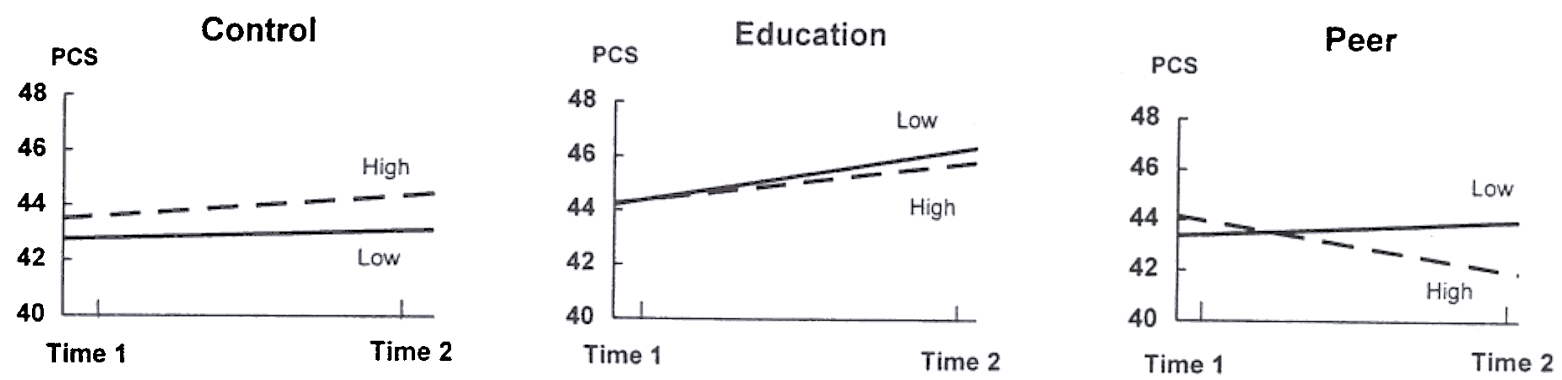

Figure 2. Interaction of baseline oncologist informational support with education and peerdiscussion interventions on PCS (physical functioning). Higher scores mean better functioning.

\section{Discussion}

Previous research has been inconclusive in demonstrating positive effects for peer support groups. In the cancer literature alone, education groups seem to be more effective than peer-discussion groups (Helgeson \& Cohen, 1996). One reason for the inconsistent findings regarding the benefits of peer-discussion groups may have to do with differential needs for emotional support. The results of this study showed that a subgroup of women benefited from the peer-discussion group - those who lacked emotional support from their partners or who conversely reported more negative interactions with their partners. In general, peer-support groups are thought to be beneficial because they compensate for deficits in one's naturally occurring network. In the instance of cancer, as well as other stressful life events, it is likely that a portion of people receive effective support from their network and do not have such deficits. These people may not benefit from a peer-discussion group.

In fact, it appears from this study that women who started out satisfied with the level of emotional support received from their partner actually deteriorated over time in physical functioning when assigned to a peer-discussion group. How could the support group harm these women? One finding from the previous report (Helgeson et al., 1999) may shed light on this issue. Earlier, we showed that negative interactions with network members increased over time for people in the peer discussion conditions relative to the no-peerdiscussion conditions (Helgeson et al., 1999). It may be that attending a peer-discussion group only adversely affected interactions with network members among those who started out satisfied with network relations.

The peer-discussion group may have altered these women's perceptions of their network relationships. Women who came to the group and perceived their network relationships to be helpful may hear stories from other group members that lead them to reevaluate their existing social relationships. For example, we had one group member who told stories about her mother-in-law at each meeting-stories

\section{Personal Resources}

Control

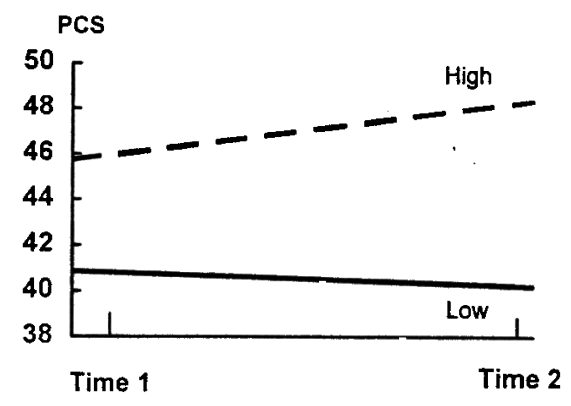

Education

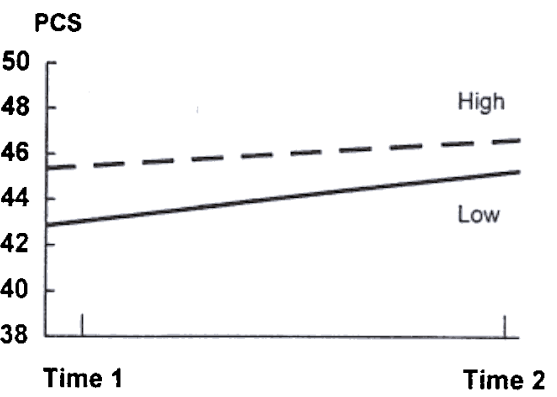

Figure 3. Interactions of baseline personal resources with education and control conditions on PCS (physical functioning). Higher scores mean better functioning. 
that most anyone would agree conveyed unhelpful and sometimes even cruel behavior. This woman always followed her stories by the statement that her mother-in-law was a kind and gracious woman. After hearing these stories for four consecutive meetings, the women in the group finally got together and told this woman that her mother-inlaw was a very mean woman. In this case, the woman's perceptions of her interactions with her mother-in-law are likely to have been altered.

Alternatively, the peer-discussion group could have increased actual negative interactions with network membersagain only among those who began the study reporting few negative interactions. Perhaps, these women had few negative interactions at home because they rarely talked about their illness. The peer-discussion intervention could have encouraged women to discuss problems more frequently at home or could have led women to discuss other people's problems. Such discussions have the potential to lead to negative interactions (e.g., someone may minimize the problem, force the person to be cheerful, discourage discussion of the illness).

In either case, it is certainly unsettling that some women may have been harmed by their assignment to the peerdiscussion group. At first glance, the clinical recommendation for peer-support groups seems clear. Health care providers should gather some information about the state of women's existing social relationships, refer those who lack support to peer-support groups, and not encourage those who are satisfied with their support to attend such groups. However, a peer-support group composed only of people who lack support may be very different from the peersupport group evaluated in this study. People who lack support have fewer social skills than other people (S. Cohen, Sherrod, \& Clark, 1986). The women with high support may have been responsible for the benefits the low support women accrued. A group of low support people may not be able to create an atmosphere of emotional support (e.g., provide validation, provide mutual aid). In short, the presence of high-support women could have contributed to the effectiveness of the peer-support group for low-support women in this study. This makes clinical recommendations more difficult. It would be worthwhile to determine whether groups of low-support persons can be effective or whether persons with high support can be trained in some way to help the low-support persons without suffering any negative consequences themselves.

Referrals to educational group interventions are much more clear. First, the overall study showed benefits of the educational intervention (Helgeson et al., 1999). In this report, we found that women who had the most difficulties at the start of the study were the most likely to benefit from the education intervention. Here, difficulties can be construed as the lack of partner emotional support, the lack of physician informational support, or the lack of personal resources (i.e., low self-esteem, low body image, low perceived control, high illness uncertainty). Recall that all of these variables were somewhat interrelated suggesting a common core of problems.

However, baseline depression as measured with the
Center for Epidemiologic Studies Depression Scale (Radloff, 1977) did not moderate the effects of the intervention. Baseline MCS revealed a marginal interaction with the educational intervention ( $p=.09$ ), suggesting that the education intervention had the most benefit on MCS for those who started out with the lowest scores. Thus, the evidence that baseline mental health moderated the effects of the interventions is weak.

One might think that social support and personal resources are correlated with a woman's socioeconomic status and that a lack of education really underlies the moderating effects reported in this article. However, neither education nor income were correlated with the independent or dependent variables in this study. In addition, education did not moderate the effects of the interventions. Disease stage also was not found to be a moderator.

The resources provided by the educational group intervention seem to meet the largest portion of women's needs. Once the materials for such an intervention are assembled, the intervention is easy to implement. Any patient could be referred to such a group intervention. Although there are differential benefits, there appear to be no costs associated with the intervention. Health care professionals could either offer the educational intervention to all women with breast cancer or minimize costs and only target the group with the lowest personal and social resources.

There are a couple of limitations to these findings. First, the results only held for PCS and not MCS. We cannot explain this disparity. Second, we collected longer term follow-up data 6 months after the intervention and found only one significant moderating effect--peer support helped people who had higher rather than lower negative interactions with partners. For the rest of the support variables, there were nonsignificant trends ( $p$ s ranged from .06 to .15) consistent with the direction of the earlier results. There was no interaction between the overall personal resources index and the educational intervention, but one component of the index (personal control) did interact with the intervention to predict PCS $(p<.05)$, such that those with the lowest perceived control received the largest benefit from the educational intervention. Thus, our findings as to who benefits from what are largely limited to the early effects of the interventions.

A remaining question is the extent to which these results generalize to other populations-to people with other health problems, to people of a much lower socioeconomic status, to minority populations, and to men. Only future research can address these questions. We see no reason why these results should not transfer to other health problems, but it may depend on the support needs associated with the problem. The support needs of minority populations have rarely been investigated, and few support interventions have focused on men. Some would argue that men have greater desires for informational support than emotional support. Does this mean that men would benefit more or less from emotional support? The answer is unclear because men are more likely than women to have emotional support deficits (Schumaker \& Hill, 1991). Support intervention work is 
underway with men with prostate cancer to evaluate this issue (Lepore, Helgeson, \& Schulz, 1999).

\section{References}

Andersen, B. L. (1992). Psychological interventions for cancer patients to enhance quality of life. Journal of Consulting and Clinical Psychology, 60, 552-568.

Bourgeois, M. S., Schulz, R., \& Burgio, L. (1996). Interventions for caregivers of patients with Alzheimer's disease: A review and analysis of content, process, and outcomes. International Journal of Aging and Human Development, 43(1), 35-92.

Campbell, S. M., Dunkel-Schetter, C. A., \& Peplau, L. A. (1991). Perceived control and adjustment to infertility among women undergoing in vitro fertilization. In A. L. Stanton \& C. A. Dunkel-Schetter (Eds.), Infertility: Perspectives from stress and coping research (pp. 133-155). New York: Plenum.

Coates, D., \& Winston, T. (1983). Counteracting the deviance of depression: Peer support groups for victims. Journal of Social Issues, 39, 169-194.

Cohen, J., \& Cohen, P. (1983). Applied multiple regression/ correlation analysis for the behavioral sciences (2nd ed.). Hillsdale, NJ: Erlbaum.

Cohen, S., Sherrod, D. R., \& Clark, M. S. (1986). Social skills and the stress protective role of support processes. Journal of Personality and Social Psychology, 50, 963-973.

Collins, R. L., Taylor, S. E., \& Skokan, L. A. (1990). A better world or a shattered vision? Changes in life perspectives following victimization. Social Cognition, 8, 263-285.

Cowan, C. P., \& Cowan, P. A. (1986). A preventive intervention for couples becoming parents. In C. F. Z. Boukydis (Ed.), Research on support for parents and infants in the postnatal period (pp. 225-251). New York: Ablex.

Dakof, G. A., \& Taylor, S. E. (1990). Victim's perceptions of social support: What is helpful from whom? Journal of Personality and Social Psychology, 58, 80-89.

Dunkel-Schetter, C. (1984). Social support and cancer: Findings based on patient interviews and their implications. Journal of Social Issues, 40, 77-98.

Edgar, L., Rosberger, Z., \& Nowlis, D. (1992). Coping with cancer during the first year after diagnosis. Cancer, 69(3), 817-828.

Fawzy, F. I., Fawzy, N. W., Anndt, L. A., \& Pasnau, R. O. (1995). Critical review of psychosocial interventions in cancer care. Archives of General Psychiatry, 52, 100-113.

Helgeson, V. S. (1992). Moderators of the relation between perceived control and adjustment to chronic illness. Journal of Personality and Social Psychology, 63, 656-666.

Helgeson, V. S., \& Cohen, S. (1996). Social support and adjustment to cancer: Reconciling descriptive, correlational, and intervention research. Health Psychology, 15, 135-148.

Helgeson, V. S., Cohen, S., Schulz, R., \& Yasko, J. (1999). Effects of education and peer discussion group interventions on 6-month adjustment to stage I and II breast cancer. Archives of General Psychiatry, 56, 340-347.

Hughes, R. J. (1988). Divorce and social support: A review. Journal of Divorce, $11(3 / 4), 123-145$.

Lavoie, J.-P. (1995). Support groups for informal caregivers don't work! Refocus the groups or the evaluations? Canadian Journal of Aging, 14(3), 580-595.

Lepore, S. J., Helgeson, V. S., \& Schulz, R. (1999). [Adjustment to prostate cancer] Unpublished raw data.

Meyer, T. J., \& Mark, M. M. (1995). Effects of psychosocial interventions with adult cancer patients: A meta-analysis of randomized experiments. Health Psychology, 14, 101-108.

Mishel, M. H. (1981). The measurement of uncertainty in illness. Nursing Research, 30(5), 258-263.

Neuling, S. J., \& Winefield, H. R. (1988). Social support and recovery after surgery for breast cancer: Frequency and correlates of supportive behaviors by family, friends and surgeon. Social Science and Medicine, 27, 385-392.

Newell, D. J. (1992). Intention-to-treat analysis: Implications for quantitative and qualitative research. International Journal of Epidemiology, 21, 837-841.

Peters-Golden, H. (1982). Breast cancer: Varied perceptions of social support in the illness experience. Social Science and Medicine, 16, 483-491.

Radloff, L. S. (1977). The CES-D scale: A self-report depression scale for research in the general population. Applied Psychological Measurement, 1, 385-401.

Rosenberg, M. (1965). Society and the adolescent self image. Princeton, NJ: Princeton University Press.

Schag, C. A. C., \& Heinrich, R. L. (1988). CARES: Cancer Rehabilitation Evaluation System. Santa Monica, CA: CARES Consultants.

Shumaker, S. A., \& Hill, D. R. (1991). Gender differences in social support and physical health. Health Psychology, 10, 102-111.

Trijsburg, R. W., van Knippenberg, F. C. E., \& Rijpma, S. E. (1992). Effects of psychological treatment on cancer patients: A critical review. Psychosomatic Medicine, 54, 489-517.

Vaux, A., Riedel, S., \& Stewart, D. (1987). Modes of social support: The social support behaviors (SS-B) Scale. American Journal of Community Psychology, 15, 209-237.

Wandersman, L. P. (1982). An analysis of the effectiveness of parent-infant support groups. Journal of Primary Prevention, 3(2), 99-115.

Ware, J. E., Kosinski, M., \& Keller, S. D. (1995). SF-36 physical and mental health summary scales: $A$ user's manual. Boston: The Health Institute.

Ware, J. E., Snow, K. K., Kosinski, M., \& Gandek, B. (1993). SF-36 Health Survey: Manual and interpretation guide. Boston: Nimrod.

Watson, M. (1983). Psychosocial intervention with cancer patients: A review. Psychological Medicine, 13, 839-846.

Wells, K. B., Stewart, A., Hays, R. D., Burnam, M. A., Rogers, W., Daniels, M., Berry, S., Greenfield, S., \& Ware, J. (1989). Detection of depressive disorder for patients receiving prepaid or fee-for-service care: Results from the Medical Outcomes Study. Journal of the American Medical Association, 262, 914-919.

Wortman, C. B., \& Lehman, D. R. (1985). Reactions to victims of life crises: Support attempts that fail. In G. Sarason \& B. R. Sarason (Eds.), Social support: Theory, research and applications (pp. 463-489). Dordrecht, The Netherlands: Martinus Nijhoff. 